\title{
DE LA CONCENTRACIÓN A LA DESCENTRALIZACIÓN DE LOS PARQUES INDUSTRIALES EN EL ESTADO DE MÉXICO, ¿ALTERNATIVA DE DESARROLLO REGIONAL?
}

\section{FROM THE CONCENTRATION TO THE DECENTRALIZATION OF THE INDUSTRIAL PARKS IN THE STATE OF MÉXICO, ¿ALTERNATIVE OF REGIONAL DEVELOPMENT?}

David Iglesias Piña, Ph.D. Doctor en Economía por la Universidad Nacional Autónoma de México (UNAM). Profesor-investigador y Coordinador del Doctorado en Sustentabilidad para el Desarrollo, Centro de Estudios e Investigación en Desarrollo Sustentable de la Universidad Autónoma del Estado de México. Toluca, Estado de México, México. diglesiasp@uaemex.mx

\section{ARTÍCULO DE REFLEXIÓN}

Recibido: 1 de agosto de 2019.

Aceptado: 23 de agosto de 2019.

\section{RESUMEN}

El proceso de industrialización vía parques industriales que experimentó el Estado de México pasó por varias fases, similar a la experimentada a nivel nacional, que buscó tanto su descentralización de la Zona Metropolitana de la Ciudad de México (ZMCM), la diversificación de la estructura productiva, así como de una mayor conectividad hacia la región centro del país. El objetivo de este artículo es analizar si el proceso de descentralización de los parques industriales constituye una alternativa para el fomento del desarrollo regional en el Estado de México. Para ello se utilizó el método de análisis histórico, a través de la revisión de las diversas iniciativas de políticas públicas, para conocer la pertinencia de dichas acciones. Los hallazgos refieren que tales acciones gubernamentales se tradujeron en una mayor polarización regional, ya que se formaron

ISSN: 1390-9320, Vol. 6, No. 5, octubre 2019 
regiones de reconcentración industrial $(R R I)$, clara expresión de la heterogeneidad territorial, productiva e industrial.

Palabras clave: parques industriales, descentralización industrial, desarrollo regional, reconcentración industrial.

\section{ABSTRACT}

The process of industrialization to industrial parks experienced by the State of Mexico went through several phases, similar to that experienced at the national level, which sought both decentralization of the Metropolitan Area of Mexico City (ZMCM), diversification of the productive structure, as well as greater connectivity to the central region of the country. The objective of this paper is analyze whether the process of decentralization of industrial parks is an alternative to promote regional development in the State of Mexico. For this was used the historical analysis method, through the revision of the diverse public policy initiatives, in order to know the pertinence of such actions. The findings refer that such governmental actions resulted a regional polarization, for the regions of industrial reconcentration (RRI), that is a clear expression of territorial, productive and industrial heterogeneity.

Keywords: industrial parks, industrial decentralization, regional development, industrial reconcentration.

\section{INTRODUCCIÓN}

El proceso de industrialización y construcción de parques industriales que se dio en México a partir de los años cuarenta, fue casi paralela a la del Estado de México, incluso fue una de las primeras entidades de la región centro del país que se incorporó de manera acelerada a esta dinámica productiva (Rózga e Iglesias, 2004), situación que favoreció la constitución de una estructura industrial más diversificada, con la pretensión elevar la productividad y el nivel de vida de la población (Martínez, 1985).

A partir de los años cuarenta, la industrialización mexiquense pasó por varias fases, similar a la experimentada a nivel nacional. Por ello, es posible identificar cuatro principales etapas,

ISSN: 1390-9320, Vol. 6, No. 5, octubre 2019 
iniciando con la industrialización moderna, seguido de la industrialización media, posteriormente la avanzada o madura y culminando con la etapa de expansión y reestructuración industrial, que comprende de 1991 y que aún sigue en proceso. En cada una de estas, se expandió tal actividad, se diversificó la estructura productiva y se crearon nuevos espacios industrializados a través de la construcción de parques industriales, concentrándose mayoritariamente en los municipios aledaños a la Zona Metropolitana de la Ciudad de México y Toluca. Acompaño de este dinamismo, también se conformaron las regiones de reconcentración industriales $(R R I)$, situación que acentuó la desigualdad entre las regiones mexiquenses, lo que significó una mayor polarización económica, social y territorial en la entidad mexiquense.

\section{REVISIÓN TEÓRICA}

\section{El contexto de los parques industriales: algunos referentes.}

El desarrollo de la industria no solo está en función de la política pública, de los programas de inversión que puede ejercer el estado; sino también de la capacidad de organización y ordenación territorial, mediante diferentes modelos organizativos como el de los parques industriales. El primer modelo de ordenamiento de la industria se suscitó en los Estados Unidos, a finales del siglo XIX donde se utilizó el término parque industrial, el cual se asoció a un espacio abierto con presencia de zonas verdes y una mayor calidad urbanística y ambiental, respondiendo las necesidades de diseño y criterios de flexibilidad y calidad técnica en servicios de infraestructura (Alonso, 1991). A partir de ello, no solo se evidenció la posibilidad de agrupar a las empresas en determinados territorios, sino también creció el interés por explicar y argumentar teóricamente el comportamiento de dichas concentraciones. Por ejemplo, Weber (1929) consideró al parque industrial como una concentración de empresas, localizadas estratégicamente en un territorio y con factores geográficos pertinentes para que pudieran operar sin problemas.

Por su parte, Lösch (1954) se centró en sostener que estos son aglomeraciones de empresas que se presentaban en determinados territorios con ciertos factores de producción para su funcionamiento. Bengston y Van Royen (1956) lo concibieron como un 
espacio físico de dimensiones adecuadas donde se agrupan varias empresas para formar una industria.

En otras concepciones, se consideran e incluyen un conjunto de nuevos elementos que hacen alusión no solamente la agrupación de empresas, sino la organización y condiciones específicas que debe tener el espacio donde éstas se aglomeran. La ONUDI (1979) por ejemplo lo concibe como un terreno urbanizado y subdividido en parcelas, dotado de carreteras, medios de transporte y servicios públicos, que pueden contar con fábricas construidas (anticipadamente), con servicios e instalaciones colectivas, destinado al uso de una comunidad industrial. Esta concepción se tomó como base para realizar planteamientos más contemporáneos, que para el caso de México sobresale el emitido por el INEGI (1999), el cual considera al parque industrial como un área planeada que permite promover el establecimiento de empresas industriales, mediante la dotación anticipada de infraestructura, naves y servicios comunes que operan bajo una administración permanente.

Similar a esta, con una perspectiva de organización industrial y de orientación regional, fue la que emitió la Secretaría de Economía del Gobierno Mexicano (2015), a través de la Norma Oficial Mexicana de Parques Industriales, considerándolo como una superficie geográficamente delimitada y diseñada especialmente para el asentamiento de la planta industrial en condiciones adecuadas de ubicación, infraestructura, equipamiento y de servicios, con una administración permanente para su operación, buscando el ordenamiento de los asentamientos industriales y la desconcentración de las zonas urbanas y conurbadas, haciendo un uso adecuado del suelo, proporcionando condiciones idóneas para que la industria opere eficientemente y se estimule la creatividad y productividad dentro de un ambiente confortable. Además de coadyuvar a las estrategias de desarrollo industrial de una región (SE, 2005). Por su parte, la Asociación Mexicana de Parques Industriales Privados (AMPIP, 2010), los asume no solo son conglomerados de empresas y espacios de producción, sino sitios con un conjunto de condiciones extraeconómicas como áreas verdes, políticas de operación internas, imagen urbana interna y externa, entre otros, que propician el pleno desenvolvimiento de las empresas y de la propia economía local. 
A partir de estas concepciones, un parque industrial es un espacio geográfico, delimitado con condiciones internas necesarias y adecuadas en términos de infraestructura y equipamiento, así como de condiciones externas capaces no solo de favorecer el establecimiento y funcionamiento de empresas, sino de generar efectos positivos a nivel local y regional.

\section{El devenir de los parques industriales en el Estado de México y la emergente descentralización}

El proceso de industrialización vía parques industriales fue uno de los principales mecanismos que se adoptó tanto en México como en el Estado de México, para transitar hacia un modelo de desarrollo compartido mediante la diversificación de la estructura productiva, la conectividad espacial y la convergencia regional. Todo ello, a través de la intervención del Estado, cuyas políticas públicas y de inversión, buscaban potencializar la capacidad productiva interna para reducir las desigualdades socioeconómicas. Sin embargo, estas expectativas fueron inalcanzables, ya que si bien se fomentó la descentralización de la industria, estos se concentraron en los municipios conurbados a la Zona Metropolitana de la Ciudad de México (ZMCM) y de Toluca (ZMCT), generando un ambiente de reconcentración y polarización socioeconómico-territorial, misma que se fue acentuando en el devenir de la industrialización estatal, iniciando con la industrialización moderna, seguido de la industrialización media, posteriormente la avanzada o madura y culminando con la etapa de expansión y reestructuración industrial, que comprende de 1991 y que aún está en proceso.

\section{Etapa de industrialización moderna, 1941-1960.}

Esta inició con el decreto de la Ley de Protección a las Industrias Nuevas y Necesarias de 1941, que buscó atraer nuevos capitales para crear fuentes de producción y de trabajo fuera de la Ciudad de México (CdMx), a través de la construcción de vías de comunicación y de infraestructura urbana, orientadas a facilitar el flujo de factores productivos y de mercancías hacia diversos mercados regionales. A pesar de haberse mejorado las condiciones infraestructurales, la mayor parte de las empresas se concentraron en los municipios mexiquenses aledaños a la CdMx, destacando Tlalnepantla, Naucalpan, Cuautitlán Izcalli y Tultitlán, con predominio de la industria textil, alimentos y bebidas, fibras, hilos, cartón e industria de la madera (Rózga e Iglesias, 2004).

ISSN: 1390-9320, Vol. 6, No. 5, octubre 2019 
Entre 1946 y 1950, el asentamiento creciente de empresas en dichos municipios propició el agotamiento de suelo industrial, por lo que fue necesario expropiar nuevos espacios para no interrumpir el avance y desarrollo de tal actividad (GEM, 1951). Para ello, entre 1951 y 1957 se realizaron adiciones a la Ley de Protección a las Industrias Nuevas y Necesarias, para exceptuar el pago de impuestos a los empresarios que estaban pagando intereses por concepto de créditos solicitados, fomentar y capitalizar la industria, así como estimular su expansión hacia otros municipios (Bejar y Casanova, 1970).

En el periodo de 1958 a 1963, dicha ley también contempló el otorgamiento de franquicias de compensación a las empresas para la ocupación de los terrenos expropiados, readecuar sus naves industriales y mejorar su sistema de producción, así fomentar el desarrollo de actividades que no existían en la entidad, como las automotrices (GEM, 1963). Este conjunto de acciones permitió la atracción y establecimiento de tres plantas del ramo referido en el corredor industrial Toluca-Lerma, a saber: Daimler-Chrysler, Carrocerías Toluca (CATOSA) y Robert Bosch, cuya especialización en el ensamble de automóviles (PT Cruiser) y cajas para ensamble, así como en la fabricación de carrocerías, alternadores, marchas y motores de enfriamiento, contribuyó a la diversificación de la estructura industrial en territorio mexiquense.

\section{Etapa de industrialización media, 1961-1975}

Fue con el establecimiento de las grandes automotrices, como inició la etapa media de industrialización estatal, caracterizado por el fortalecimiento de las industrias ya existentes y el establecimiento de nuevas empresas en algunos municipios semiurbanos y rurales, con el fin de acoplarse a la tendencia del desarrollo industrial nacional, siguiendo los principios del programa nacional de descentralización y construcción de parques industriales.

Construcción de los primeros parques industriales.

En esta etapa fue decisiva la consolidación de la industria mexiquense, pues se fomentó el desarrollo de las industrias ya existentes, se crearon las condiciones para el establecimiento de nuevas plantas y se propició una ubicación ordenada de las empresas, que no solo contribuyó a mejorar la estructura productiva estatal, sino también se posicionó como la segunda entidad más importante del país, sólo por debajo de la Ciudad de México (CdMx) en el desarrollo de la industria extractiva y de transformación. Bajo esta connotación, el

ISSN: 1390-9320, Vol. 6, No. 5, octubre 2019 
Estado de México se convirtió en uno de los polos de atracción económica más relevantes a nivel nacional. A nivel regional, mostró su fortaleza y liderazgo tanto industrial como económico, convirtiéndose en uno de los principales mercados y centros de producción, compitiendo fuertemente con la CdMx.

Para mantener esta posición, la política económica centró su atención en la creación de cinco áreas geoeconómicas o corredores industriales prioritarios, tal como se aprecia en la Figura 1. Dichos corredores se integraron por los siguientes municipios (Rózga e Iglesias, 2004):

1) El Oro-Atlacomulco-Ixtlahuaca.

2) La Marquesa- Santiago Tianguistenco-Lerma.

3) Ocoyoacac-Lerma-Toluca.

4) Villa Victoria-Zinacantepec-Almoloya de Juárez.

5) Zumpango-Huehuetoca.

Con esta configuración territorial, se buscó fomentar tanto el crecimiento de la actividad en comento, como influir en el surgimiento de nuevos espacios económicos locales y regionales para dinamizar el desarrollo de la entidad mexiquense, sobre todo por qué en la mayoría de los municipios que conformaron estos nuevos nodos económicos, se construyeron los primeros parques industriales, con la intensión de propiciar un ambiente más organizado de tal actividad y la consecución de la política de descentralización industrial y desarrollo regional.

ISSN: 1390-9320, Vol. 6, No. 5, octubre 2019 
Figura 1. Formación de corredores industriales en el Estado de México.

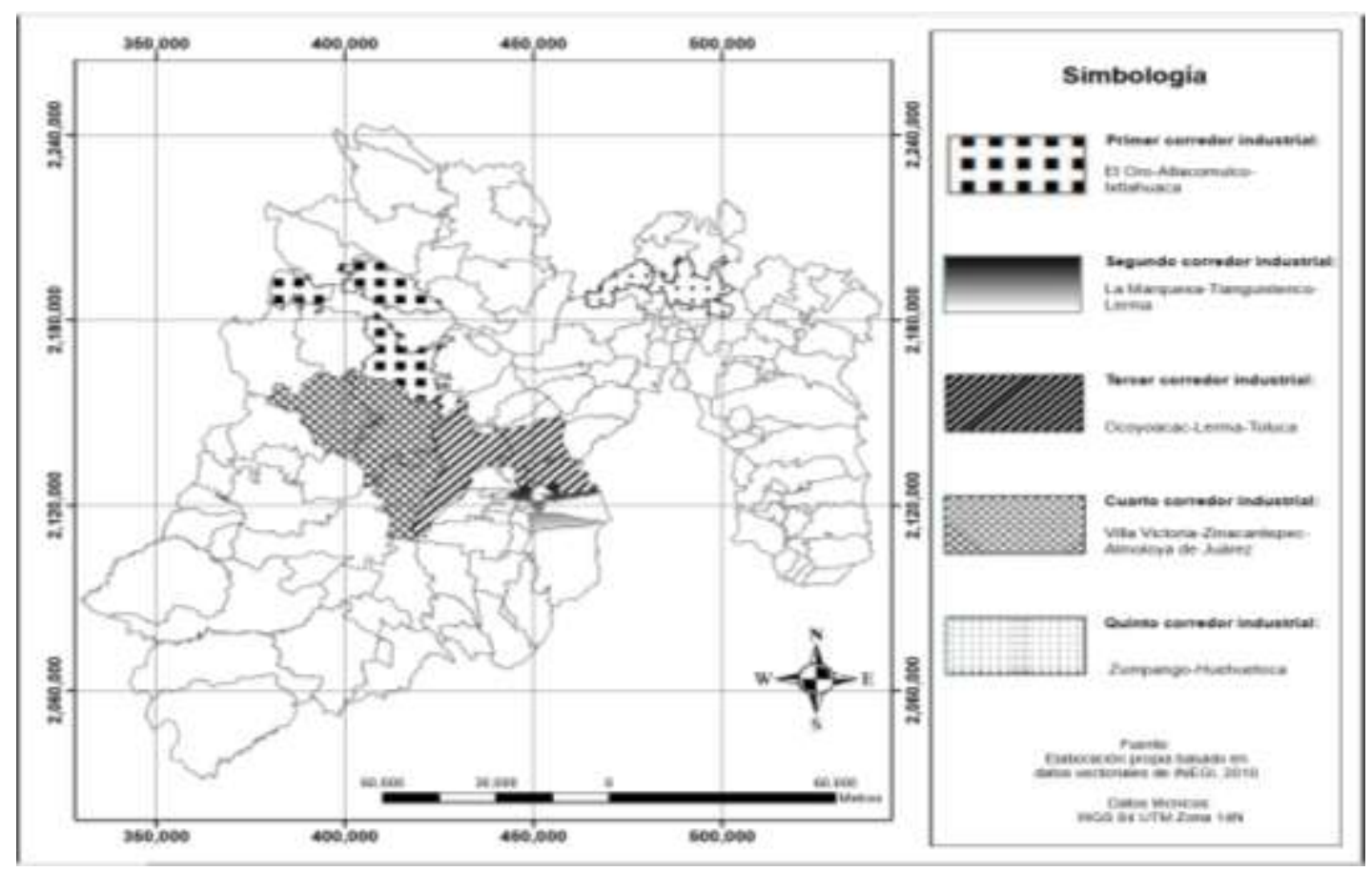

Nota: Espacio para notas aclaratorias o información adicional de la tabla.

A pesar de la creación de estos nuevos corredores industriales para ampliar la base productiva, no todos los municipios tuvieron las condiciones suficientes, en términos de infraestructura y equipamiento urbano industrial, para dar cabida a los parques industriales y garantizar la operatividad de las empresas. Por ello, el cuarto corredor integrado por Villa Victoria, Zinacantepec y Almoloya de Juárez, no fue favorecido con ningún espacio de este tipo, y sólo el último municipio fue incorporado en el programa "ciudades modelo" implementado por el gobierno estatal en el año 2007 para crear zonas habitacionales y centros comerciales, cuyos beneficios han sido marginales (empleos temporales), opacados por la modificación de usos de suelo (de agrícola a habitacional y comercial), el encarecimiento y racionamiento del agua potable (principalmente para la población local), la ruptura de las costumbres y tradiciones locales, así como el aumento de la inseguridad derivado del gran flujo de personas en dichos asentamientos humanos.

Situación similar se presentó en Zinacantepec, que al estar conurbado a la capital del Estado de México y ser paso obligado hacia el Estado de Michoacán, se ha convertido en una "ciudad de comercio orilla", ya que solo algunos tramos de la vialidad principal se ha

ISSN: 1390-9320, Vol. 6, No. 5, octubre 2019 
desarrollado el comercio y servicio establecido. Recientemente se ha intensificado la construcción de zonas habitacionales, lo que ha fomentado el crecimiento del tercer sector propiciado por la sociedad del lugar.

En contraparte, Villa Victoria no presenta la misma dinámica. Si bien aumento el número de establecimientos comerciales y de servicios a lo largo de la carretera federal TolucaZitácuaro (Michoacán), su ubicación alejada de la Zona Metropolitana de la Ciudad de México (ZMCM), lo convirtió en un espacio poco dinámico y atractivo para la industria, incluso se sigue considerando un municipio semi-urbano de alta marginación, razón de su nula atracción industrial.

De aquí que solo los municipios con las condiciones necesarias para garantizar la funcionalidad de las empresas, se vieron favorecidos con la construcción de parques industriales, tal fue el caso de Toluca, que en 1963 se construyó el primero de estos, seguido del parque industrial Cartagena (Tultitlán) constituido en 1969, así como de tres más, distribuidos en diversos municipios. Tres de los cinco parques edificados fueron de carácter privado (Cartagena, El Trebol, y el de Tenango del Valle), en tanto que El Oro y el parque industrial de Santiago Tianguistenco fueron de inversión pública, creadas en 1972 por el Gobierno del Estado de México y el Gobierno Federal, a través del Fideicomiso de Conjuntos, Parques, Ciudades Industriales y Centros Comerciales (FIDEIN), considerada una de las políticas macroeconómicas que apoyó significativamente la construcción de dichos desarrollos industriales.

Dada la gama de estímulos, apoyos y facilidades fiscales y administrativas que ofreció el FIDEIN, se favoreció el establecimiento de 400 nuevas empresas distribuidas en 19 zonas de desarrollo industrial y 10 parques industriales, urbanizados y edificados por el Instituto de Acción Urbana e Integración Social (Instituto AURIS), como acción para intentar consolidar dichos desarrollo en el Estado de México, así como fomentar la articulación del ambiente urbano-regional con las políticas de transporte, de recursos naturales, fuerza de trabajo, migración, mercados, energéticos y dotación de infraestructura.

Relevancia económica de los parques industriales.

Uno de los primeros impactos que se esperaba se presentaría en las localidades donde se construyeron los primeros parques industriales fue la derrama de empleos, sin embargo los

ISSN: 1390-9320, Vol. 6, No. 5, octubre 2019 
resultados fueron muy heterogéneos, pues mientras en los municipios conurbados a la Zona Metropolitana de la Ciudad de Toluca (ZMCT) y de México (ZMCM) que ya contaban con cierta actividad industrial, la operatividad tanto de las empresas como de los propios parques fue mucho más dinámico que aquellos establecidos en los municipios alejados de dichas zonas conurbadas. De esta manera, el número de empleos y los efectos multiplicadores generados por los parques industriales fueron marginales en sus primeros años de funcionamiento.

Ello significó que en dicha etapa de desarrollo industrial, la estructura ocupacional sectorial no se vio significativamente influenciada por tal actividad, incluso algunos municipios como Atlacomulco, El Oro, Tenango de Valle y Santiago Tianguistenco, considerados como espacios de tradición agropecuaria, conservaron su carácter, incluso el ritmo de crecimiento ocupacional en este sector fue más acelerada que el de la industria; por tanto, las actividades agropecuarias no perdieron importancia ocupacional al menos en estos años.

Estos beneficios marginales fueron justificados por las autoridades gubernamentales, en el sentido de que como proyectos de largo plazo se requiere de un tiempo razonable para que generen efectos multiplicadores y economías de aglomeración en los entorno locales y regionales, máxime si no disponen de todas las condiciones básicas, pues varios de los componentes de la infraestructura y equipamiento urbano industrial demandan altos niveles de inversión, que no siempre el estado lo dispone y por lo tanto el nivel de atracción de dichos desarrollos se van postergando.

\section{Etapa de industrialización madura, 1976-1990}

A pesar de los esfuerzos previos de descentralización vía parques industriales, en el periodo 1976-1981, se siguió observando la concentración de dichos desarrollos hacía los municipios conurbados a la Ciudad de México (CdMx), así como en la región centro del Estado de México, específicamente en los municipios de Toluca y Lerma. Esta dinámica productiva se conoció como etapa de industrialización madura o avanzada, que abarcó de 1976 a 1990, caracterizada por la relativa pérdida de dinamismo de las actividades manufactureras, cuyos efectos permearon en la ocupación industrial, que si bien presentó tasas de crecimiento favorables, estas fueron menores respecto al sector terciario, que de 1980 a 1990 duplicó el número de personas ocupadas, mientras que la industria y los

ISSN: 1390-9320, Vol. 6, No. 5, octubre 2019 
empleos manufactureros apenas creció $60 \%$, equivalente a poco más de 300 mil empleos en este decenio.

Expansión de la construcción de parques industriales.

En este lapso, se dio continuidad a la política de (aparente) descentralización industrial, mediante el otorgamiento de facilidades administrativas y fiscales a las empresas que se reubicaran en los municipios que ya contaban con parques industriales como Lerma, Toluca, Ocoyoacac, Santiago Tianguistenco y Atlacomulco. Así como Aculco, Temascalcingo, Tenancingo y Tejupilco, considerados viables para propiciar el desarrollo de dicha actividad e incluso se catalogaron como los nodos que ampliarían los corredores industriales hacia el norte y sur de la entidad. Para ello, el gobierno estatal creó (1982-1983) el Fideicomiso para el Desarrollo de Parques y Zonas Industriales (FIDEPAR), con el objetivo de adquirir reservas territoriales en dichos municipios, a fin de propiciar el establecimiento ordenado de empresas e impulsar un desarrollo regional equilibrado. De esta manera, entre 1976 y 1990 se construyeron ocho nuevos desarrollos, con diferentes características, tal como se aprecia en la Tabla 1.

A pesar de esta expansión industrial, el Estado no se ocupó totalmente en crear la infraestructura urbana industrial necesaria para propiciar el establecimiento de empresas, situación que limitó el nivel de atracción. Ello significó que el $62.5 \%$ de los nuevos desarrollos construidos se concentró en los municipios de Toluca y Lerma, por lo que el intento de descentralización más bien se tradujo como un fenómeno de reconcentración industrial, favoreciendo mayoritariamente a las demarcaciones con tradición o base industrial (Ocoyoacac, Santiago Tianguistenco y Tenango del Valle), y marginalmente aquellos considerados como "nuevos" nodos industriales (Ixtlahuaca, Jilotepec y El Oro) ubicados en la Zona Poniente de la entidad mexiquense (ZPEM), cuya conectividad con los estados Querétaro y Michoacán se ha retardado más allá de lo estipulado en el discurso oficial del gobierno estatal y federal.

Tabla 1. Parques Industriales creados en la etapa de industrialización madura estatal, 1976-1990. 


\begin{tabular}{l|llll}
\hline $\begin{array}{l}\text { Parque } \\
\text { Industrial }\end{array}$ & $\begin{array}{l}\text { Ubicación } \\
\text { (municipio) }\end{array}$ & $\begin{array}{l}\text { Año de } \\
\text { constitución }\end{array}$ & $\begin{array}{l}\text { Extensión } \\
\text { (hectáreas) }\end{array}$ & Propiedad \\
\hline Atlacomulco & Atlacomulco & $1978-1981$ & 2920.7 & Pública \\
Toluca & Toluca & 1978 & 28.0 & Privada \\
El Coecillo & Toluca & 1980 & 170.0 & Privada \\
Huehuetoca & Huehuetoca & 1980 & 749.9 & Pública \\
(Américas) & & & & \\
El Cerrillo I & Lerma & $1982-1983$ & 382.1 & Pública \\
San Antonio & Toluca & 1983 & 140.8 & Privada \\
$\begin{array}{l}\text { Buenavista } \\
\text { Exportec I }\end{array}$ & Toluca & 1981 & 246.1 & Pública \\
Jilotepec & Jilotepec & 1987 & 312.2 & Pública \\
\hline
\end{tabular}

Fuente: Elaboración propia con base en FIDEPAR, 2009 y SIMPPI, 2010.

Conformación de nuevas regiones industriales.

La dinámica que experimentaron los parques industriales ubicados fuera de la ZMCT, principalmente hacia el norte, aunado a la conectividad vial y contigüidad con la Ciudad Industrial "Pasteje" ubicada en el municipio de Jocotitlán, el parque industrial de Ixtlahuaca que apenas estaba en proyecto, así como el área de influencia que se estableció con el parque ubicado en El Oro y el pujante desarrollo industrial de San Juan del Río, en el estado de Querétaro, permitieron configurar el corredor industrial periférico en la región norte de la entidad mexiquense, tal como se observa en la Figura 2. Dicho corredor se integró tanto por parques que habían alcanzado cierta madurez productiva como el de Atlacomulco, otros que estaban en proceso de crecimiento como el de Jilotepec, así como los que estaban en proyecto, que fueron los casos del parque industrial San Cayetano de Toluca y el de Ixtlahuaca.

Figura 2. Configuración del corredor industrial periférico norte en el Estado de México. 


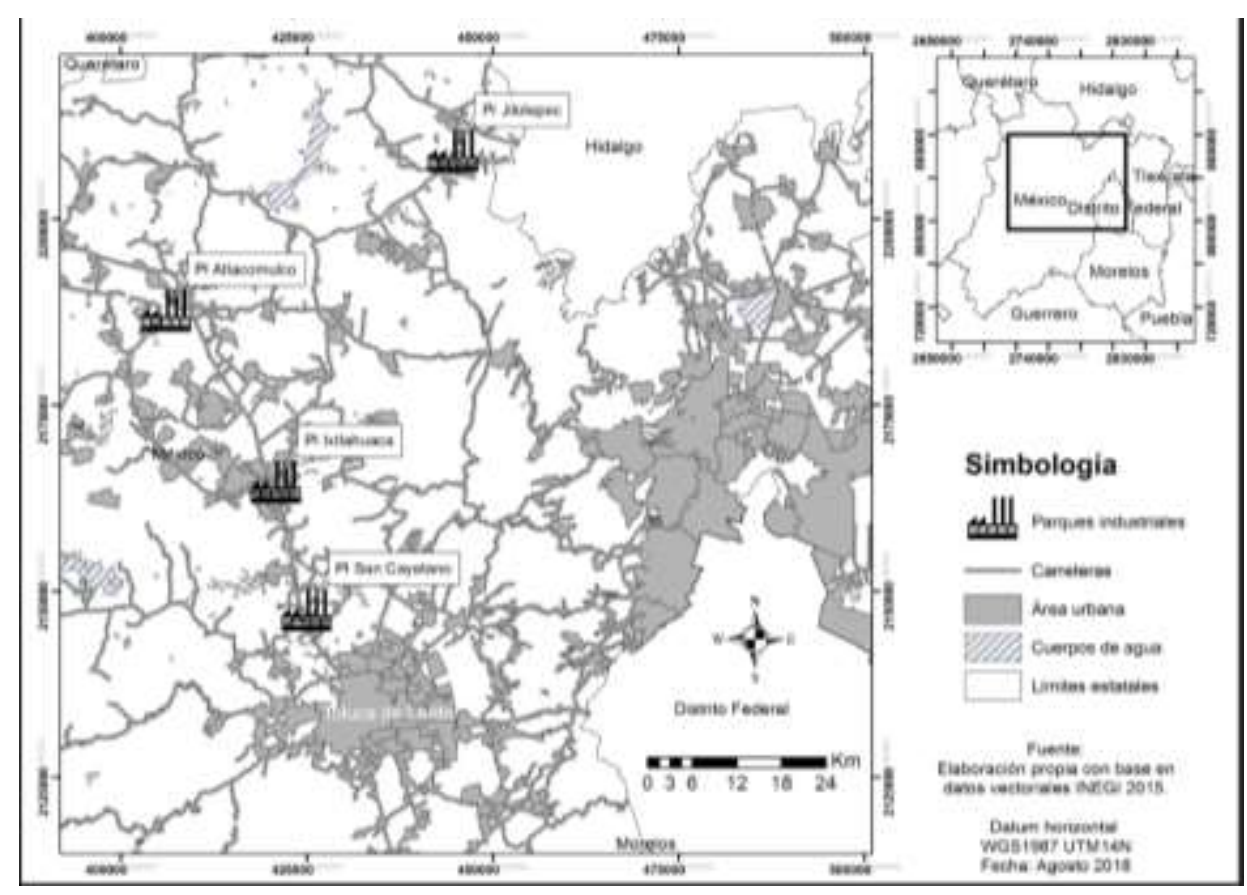

Nota: Espacio para notas aclaratorias o información adicional de la tabla.

En contraparte, el resto de los parques creados en esta etapa de industrialización madura mexiquense, dada su ubicación, tuvieron un comportamiento influenciado por la dinámica de la ZMCM y de la ZMCT, misma que les permitió aprovechar las ventajas creadas por los que ya estaban operando en dichos lugares, aunque es claro que la vecindad no es garantía de ocupación plena. Muestra de ello fueron los parques industriales El Cerrillo y Exportec I, cuya falta de ocupación total, se atribuyó a los altos costos de suelo y de arrendamiento de lotes, así como la competencia ejercida por los parques privados como el Toluca 2000 y el Coecillo I y II, que ofrecieron mejores servicios y condiciones para la adecuada operación de las empresas. Estas restricciones ocupacionales que enfrentaron dichos parques, se reflejaron en los niveles de empleo generado en sus respectivos municipios. De hecho, los impactos laborales de los parques industriales localizados en el municipio de Toluca fueron de las menos dinámicas, con una tasa de crecimiento de $36 \%$, al pasar de 5,983 puestos de trabajo en 1976 a 8,129 en 1990.

En secuencia, la mayor parte de los parques construidos en esta etapa se les definió un perfil acorde a la región donde se localizaron. La Tabla 2, muestra que la mayoría fue

ISSN: 1390-9320, Vol. 6, No. 5, octubre 2019 
considerada como de vocación manufacturera textil. Bajo esta acotación, la política pública no sólo buscó el asentamiento de nuevas empresas en dichos espacios, sino orientó su ubicación por giro principal de tal manera que permitiera formar las primeras cadenas de producción empresariales. Fue así como a finales de los años noventa se configuró el cluster industrial manufacturero, y a pesar de haber tenido un carácter incipiente dado el reducido número de empresas y la poca interacción entre estas, puede ser tomando como una de las primeras bases para la posible integración de un sistema productivo local de base industrial.

Tabla 2. Vocación productiva de los parques industriales creados en la etapa de industrialización madura, 1976-1990.

\begin{tabular}{l|ll}
\hline Parque industrial & Vocación productiva & Giro principal \\
\hline Atlacomulco & Manufacturero & Servicios y maquila \\
Toluca & Manufacturero & Maquila \\
El Coecillo & Manufacturero & Maquila \\
Huehuetoca & Manufacturero y de servicios & Servicios y cogeneración de \\
(Américas) & & energía \\
El Cerrillo I & Farmacéutico y manufacturero & Alimentos, farmacéutica y \\
& & textiles \\
San Antonio & Servicios & Servicios y distribución \\
Buenavista & & \\
Exportec I & Servicios y manufacturero & Servicios bancarios y maquila \\
Jilotepec & Química y manufacturero & Química y textil \\
\hline
\end{tabular}

Fuente: Elaboración propia con base en FIDEPAR, 2009.

\section{Etapa de expansión y reestructuración, 1991-2018.}

El proceso de expansión de la mancha metropolitana, las externalidades generadas por la excesiva aglomeración de actividades económicas y la salida de empresas hacia la periferia se concibió como etapa de expansión y reestructuración industrial, caracterizada por la creación de nuevos parques industriales hacia la ZPEM. La movilidad que experimentaron las empresas ubicadas en la ZMCM y la ZMCT hacia los márgenes del estado de México en esta etapa que inició en 1991 y que aún está en proceso, fue considerado como una oportunidad 
para que el gobierno mexiquense implementara políticas de apoyo administrativo y fiscal, con el fin de que estos se asentaran en los parques industriales ya existentes. Acompañado de esta movilidad de empresas, la Tabla 3 muestra que también se construyeron seis nuevos parques que fungieron como espacios de desarrollo de recientes unidades de producción y nodos que complementaron los corredores industriales, principalmente hacia el norte de la entidad, aunque el $50 \%$ de estos se siguieron concentrando en la zona metropolitana, principalmente en los municipios de Toluca y Lerma. Respecto al tipo de propiedad, sólo la reserva industrial Santa Bárbara de Atlacomulco fue de capital privado, el Hermandad Estado de México ubicado en la zona oriente del municipio Ixtlahuaca, de capital mixto y el resto fue construido con recursos públicos y administrados por el FIDEPAR.

Tabla 3. Parques Industriales creados en el estado de México en la etapa de expansión y reestructuración industrial, 1991-2018.

\begin{tabular}{|c|c|c|c|c|}
\hline $\begin{array}{l}\text { Parque } \\
\text { Industrial }\end{array}$ & $\begin{array}{l}\text { Ubicación } \\
\text { (municipio) }\end{array}$ & $\begin{array}{l}\text { Año de } \\
\text { constitución }\end{array}$ & $\begin{array}{l}\text { Extensión } \\
\text { (hectáreas) }\end{array}$ & Propiedad \\
\hline Exportec II & Toluca & 1991 & 671.3 & Pública \\
\hline Cerrillo II & Lerma & 1991 & 249.9 & Pública \\
\hline $\begin{array}{l}\text { Microindustrial Cuautitlan } \\
\text { Izcalli }\end{array}$ & Cuatitlán Izcalli & 1996 & 20.7 & Pública \\
\hline $\begin{array}{l}\text { Hermandad Estado de } \\
\text { México }\end{array}$ & Ixtlahuaca & 1997 & $51^{*}$ & Mixto \\
\hline San Cayetano & Toluca & 2004 & 44.2 & Pública \\
\hline Santa Barbará & Atlacomulco & 2005 & 1070.2 & Privada \\
\hline
\end{tabular}

* Antes del año 2004 tenía 32 hectáreas de extensión, pero al ampliarse e integrarse el área con la Reserva Territorial Santa Margarita de 19 hectáreas, conformó su extensión total actual.

Fuente: Elaboración propia con base en INEGI, 1999; FIDEPAR, 2009.

Perfil productivo de los nuevos parques industriales construidos.

ISSN: 1390-9320, Vol. 6, No. 5, octubre 2019 
A pesar de la reciente creación de estos parques, la mayoría proyectó buen ambiente de crecimiento, pues en general tuvieron un nivel de ocupación del $85 \%$, siendo los parques de Santa Bárbara, San Cayetano y el de Cuautitlán Izcalli los que hasta el cierre del año 2015 estaban saturados, no así para el caso de El Cerrillo y el de Ixtlahuaca, que apenas habían logrado ocupar el 56 y 25\% respectivamente de su dimensión, estimado por el número de empresas que concentraban. Al igual que en la etapa anterior, la política de construcción de parques industriales se orientó en definir la vocación de dichos espacios, como puede apreciarse en la Tabla 4, dando prioridad el establecimiento de empresas manufactureras, y así seguir ampliando y fortaleciendo los cluster manufactureros y textiles que previamente se habían formado.

La definición de la vocación de cada uno de los parques construidos también tuvo el objetivo de contribuir a la especialización industrial de la entidad, pues tanto se amplió los cluster productivos manufactureros, como se conformaron regiones plenamente identificados por su actividad, facilitando la canalización de inversiones y la aplicación de programas de apoyo a las empresas. Sin embargo, al tener un bajo nivel de ocupación, las administradoras dieron cabida a otro tipo de unidades de producción de giro diferente, propiciando perfiles industriales bifuncionales, trifuncionales, semi diversificados y diversificados. Poco fueron los especializados o unifuncionales. ${ }^{1}$ Los municipios cuyos parques industriales fueron de reciente creación y alejados de la ZMCM y la ZMCT, presentaron un carácter unifuncional y bifuncional, mientras que en aquellas demarcaciones conurbadas a las zonas metropolitanas con parques industriales maduros, tuvieron una estructura trifuncional, semi diversificada y diversificada. ${ }^{2}$

\footnotetext{
1 Desarrollo industrial especializado en una sola actividad, y bifuncional, cuando son dos actividades las significativas dentro de los desarrollos industriales.

2 En una estructura productiva trifuncional son tres las ramas de actividad industrial las que sobresalen en los parques, mientras que cuando estas son cuatro, se dice que las actividades son semi diversificadas y es diversificada cuando la vocación del parque está sustentada por más de cinco ramas de actividad industrial.
}

ISSN: 1390-9320, Vol. 6, No. 5, octubre 2019 
Tabla 4. Vocación de los parques industriales creados en el estado de México en la etapa de expansión y reestructuración industrial, 1991-2018.

\begin{tabular}{l|l|l}
\hline $\begin{array}{l}\text { Parque } \\
\text { Industrial }\end{array}$ & Vocación productiva & Giro principal \\
\hline Exportec II & Manufacturera & Textiles, servicios y farmacéutica \\
Cerrillo II & $\begin{array}{l}\text { Automotriz y } \\
\text { manufacturera } \\
\text { Microindustrial } \\
\text { Cuautitlan Izcalli }\end{array}$ & $\begin{array}{l}\text { Automotriz, alimentos, farmacéutica, } \\
\text { textil }\end{array}$ \\
$\begin{array}{l}\text { Hermandad Estado } \\
\text { de México }\end{array}$ & Manufacturera & Servicios \\
San Cayetano & $\begin{array}{l}\text { Manufacturera y } \\
\text { servicios }\end{array}$ & Alimentos y servicios \\
Santa Barbará & Manufacturera & Plásticos y combustibles \\
\hline
\end{tabular}

Fuente: Elaboración propia con base en INEGI, 1999; FIDEPAR, 2009.

Asimismo, varios de los parques construidos en la etapa media de industrialización fueron los que se especializaron e incluso han mantenido ese perfil a lo largo del tiempo, mientras que los de reciente creación, la mayoría ha dado cabida a empresas de giro múltiple.

Esta estructura productiva, fue reflejo de una especialización industrial muy polarizada en tres subsectores de la actividad manufacturera, pues en conjunto concentraron más del $70 \%$ de la producción del sector, siendo éstos: productos metálicos, maquinaria y equipo; alimentos, bebidas y tabaco; y sustancias químicas, derivados del petróleo y plásticos. Los municipios mexiquenses con carácter metropolitano concentraron más del $90 \%$ de este potencial estatal, derivado de los grandes asentamientos de empresas; mientras que el restante $10 \%$ se distribuyó en los municipios periféricos a la metrópoli. 
La contrariedad de la descentralización: la reconcentración de los parques industriales.

El 62\% de los parques industriales que existían en la entidad a principios del año 2018, estaban concentrados al nororiente y norponiente del estado de México, es decir, en los municipios conurbados a la Ciudad de México; mientras que el $28 \%$ se distribuyó en la región centro de la entidad mexiquense y el restante $10 \%$ en la zona poniente, donde se formó el corredor industrial periférico norte. Frente a esta asimetría, si bien la construcción de parques industriales fomentó el desarrollo de la industria, este no fue con las mejores condiciones, pues la excesiva concentración de estos propició un patrón industrial en forma de anillos (Rózga, 1992), aunque en estricto sentido al no cumplirse el criterio de continuidad y contigüidad territorial, tal como se observa en la Figura 3, no pueden considerarse como tal, más bien por su distribución geográfica son regiones de reconcentración industrial (RRI), concebidos como espacios donde ya estaban establecidas algunas empresas con condiciones infraestructurales necesarias para favorecer el desarrollo de actividades industriales, mismas que se convirtieron en atractivos de las empresas que se habían ubicado en otros municipios. Por lo tanto, las empresas que inicialmente se establecieron en algunos parques industriales ubicados en otros municipios alejados de la ZMCM y ZMCT, retornaron a estos lugares, generando un fenómeno de reconcentración creciente de empresas y de la propia industria.

Figura 3. Regiones de reconcentración industrial (RRI) en el Estado de México.

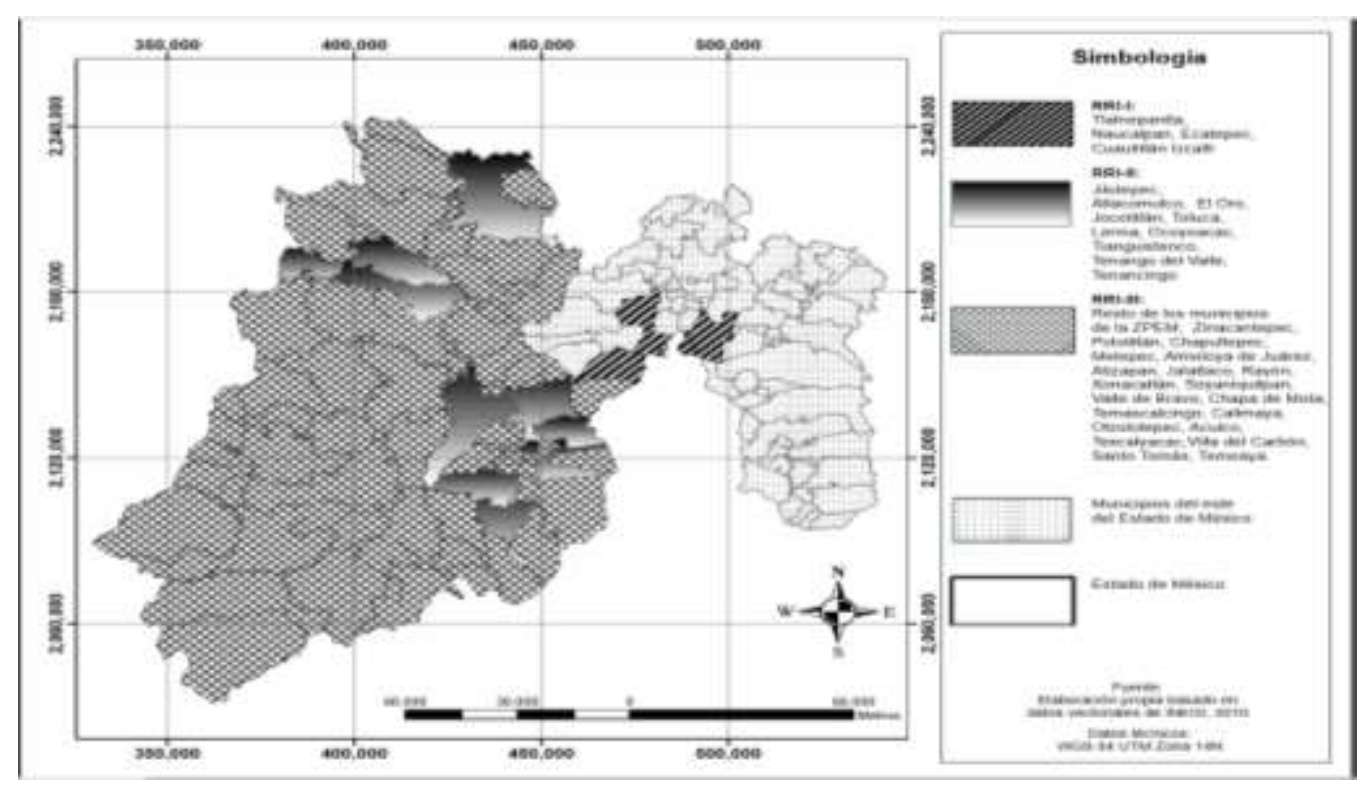

Nota: Espacio para notas aclaratorias o información adicional de la tabla.

ISSN: 1390-9320, Vol. 6, No. 5, octubre 2019 
Bajo estos argumentos, se han identificado tres RRI:

La primera integrada por los municipios de Tlalnepantla, Naucalpan, Ecatepec y Cuautitlán Izcalli, que están conurbados a la Ciudad de México, que de manera natural representan la extensión territorial del desarrollo industrial metropolitano. Estos se caracterizan por una estructura productiva diversificada, fundamentalmente manufacturera. En esta región, sigue dominando la actividad industrial, observada prácticamente en todos los aspectos (número de establecimientos, población ocupada, valor agregado, capital fijo), aunque cada vez más pierde su preeminencia en favor de la segunda región. Las industrias aquí ubicadas, son en gran parte modernas, pero su grado de modernidad, medido, por ejemplo, a través del indicador de capital por trabajador, es en promedio más bajo, que el de las industrias de la región dos.

La segunda está conformada por los municipios que integran la Zona Poniente del Estado de México (ZPEM), siendo algunos de estos Jilotepec, Atlacomulco, El Oro, Jocotitlán, Toluca, Lerma, Ocoyoacac, Santiago Tianguistenco y Tenango del Valle. Esta región no se ha terminado de formar completamente, se encuentra en expansión dado los recientes parques industriales construidos, por lo que está tomando mayor importancia, no solo en términos económicos, sino también territoriales, por la formación del corredor industrial periférico norte.

La tercera se integra por los municipios que se encuentran a una mayor distancia de la ZMCM y de la ZMCT. En éstos aparecen, de manera puntual sólo algunas industrias pequeñas, rara vez medianas, básicamente agroindustrias o industrias alimenticias. Incluso, en muchos casos se trata más bien de talleres familiares de carácter agroindustrial, que abastecen a la población local de algunos productos de primera necesidad como panaderías, tortilleras, producción de sandalias, prendas de vestir, entre otros.

Al realizar un ejercicio regional más sintético, tomando como criterio el lugar de ubicación actual de los desarrollos industriales, es posible conformar cuatro grandes bloques con diferentes niveles de consolidación industrial, y que de alguna manera guardan relación con las RRI identificadas.

El primer bloque se integra por los municipios localizados al nororiente de la entidad, mismas que están conurbadas a la ZMCM y que aglutinan el 38.6\% de los desarrollos industriales, distribuidos en siete municipios: Tlalnepantla, Ecatepec, Tultitlán, Nezahualcóyotl, Ixtapaluca,

ISSN: 1390-9320, Vol. 6, No. 5, octubre 2019 
Valle de Chalco y Chalco. En esta zona, operaban alrededor de 1,240 empresas, que emplean cerca de 62 mil personas de dichos municipios, donde el 30.6\% estaba inserta en la industria manufacturera. Ello significó que los parques industriales generaron el $32.4 \%$ del empleo total, equivalente al $8.1 \%$ del empleo estatal.

En este grupo de municipios, destaca Tlalnepantla al concentrar 17 de los 40 desarrollos localizados en dicha agrupación territorial, dando empleo a 31,866 personas, equivalente al $51.4 \%$ de este conjunto espacial, mismas que se encuentran ocupados en el Centro Industrial Tlalnepantla, PI Barrientos, La Loma, San Nicolás Tlaxcolpan, Puente de Vigas, Las Armas, San Pablo Xalpa, Los Reyes y el parque San Lorenzo, desarrollando actividades propias de la industria manufacturera.

El segundo bloque de municipios se integra por Naucalpan, Cuautitlán Izcalli, Tepotzotlán, Atizapán de Zaragoza, Cuatitlán de Romero Rubio, Huehuetoca, Coacalco, Tecámac y Axapusco, localizados al norponiente de la entidad. Al igual que el grupo anterior, están establecidos 31 desarrollos industriales, empleando al $29.8 \%$ de la población ocupada de este conjunto municipal, equivalente al $3.7 \%$ del empleo estatal, al $16.3 \%$ de la ocupación industrial y al $23.3 \%$ de la fuerza de trabajo manufacturera de la entidad, ocupadas en cualquiera de las 1,159 unidades de producción que existen en los diferentes desarrollos industriales. Cuautitlán Izcalli es el municipio más relevante por el número de aglomeraciones concentradas ( 11 de los 31 localizados en el bloque), generando 24,678 empleos, creadas por las 594 empresas localizadas en los parques industriales de Cuamatla, Cuautitlán Izcalli Xhala, La Luz, Cuautitlán Izcalli, San Sebastían Xhala, entre los más relevantes. Asimismo, en Naucalpan de Juárez, se encuentran 6 desarrollos, que dan empleo a cerca de 22 mil personas insertas en las 500 unidades de producción industrial localizadas en los parques industriales La Perla, Atoto, Naucalpan, Naucalpan $2^{\underline{a}}$ y $3^{\underline{a}}$ secciones; así como en el conjunto industrial Tlatilco. De esta ocupación, el $69.5 \%$ desarrolla alguna labor manufacturera, sobresaliendo la fabricación de artículos de plástico, metálicos, y de fibras blandas. Ello significó que, entre ambos municipios generaron el $70 \%$ del empleo en este grupo de municipios y concentraron poco más del $80 \%$ de las unidades económicas existentes.

El tercer bloque está integrado por 7 municipios, sobresaliendo Toluca, Lerma, Santiago Tianguistenco, Tenango del Valle, San Antonio la Isla, Ocoyoacac y Capulhuac, que en conjunto aglutinaron a 25 desarrollos industriales, conformada por 734 empresas que

ISSN: 1390-9320, Vol. 6, No. 5, octubre 2019 
generaron 52,551 empleos, lo que representó el 2.2\% y el 2.5\% de las unidades de producción y empleo estatal respectivamente. En este grupo, Toluca asumió el papel de líder tanto por el número de parques industriales como de empresas existentes y empleos generados. Dicha demarcación cuenta con 11 desarrollos, empleando a 36,260 personas dentro de las 601 empresas establecidas en los parques industriales de San Antonio Buenavista, Toluca, Toluca 2000 y Exportec I y II. Este municipio generó el 69\% del empleo y aglomeró el $81.9 \%$ de las empresas existentes en dicho bloque.

El último bloque de municipios se ubica al norte de la entidad, sobresaliendo El Oro, Jilotepec, Ixtlahuaca, Atlacomulco, Jocotitlán y Polotitlán, cuyas características económicas y productivas lo convierten en un espacio muy heterogéneo, sobre todo porque varios de los desarrollos industriales establecidos no fueron del todo atractivos, dada la disponibilidad limitada de infraestructura y equipamiento urbano industrial. A pesar de esta restricción infraestructural, se consideró como una región con gran potencial de crecimiento, sobre todo por la disponibilidad de lotes industriales y la cercanía geográfica con los estados de Querétaro, Guanajuato y Michoacán, que además de ampliar la franja industrial suroeste del país, constituyó un importante mecanismo para crear redes de producción inter empresariales, que son la base fundamental de los sistemas productivos locales.

Esta heterogeneidad regional aunado a su perfil productivo, son dos elementos que explican su relevancia ocupacional en cada uno de los municipios donde están asentados. En términos generales, el empleo que generaron los parques industriales fue positiva en todos los municipios, con una tasa de crecimiento de $16.3 \%$ durante el periodo, equivalente al $29 \%$ de la ocupación manufacturera y $18 \%$ del sector industrial. Esto es indicio de la importancia que juegan dichos espacios y que constituye el respaldo de la política estatal en esta materia. Al analizar este comportamiento para cada uno de los parques, se visualizan conductas muy polarizadas, pues existen algunos cuyos niveles ocupacionales son crecientes, tal es el caso de Jilotepec, Ixtlahuaca, San Cayetano y Ocoyoacac, que en promedio contribuyeron con el $16 \%$ del empleo municipal, el $21 \%$ de la ocupación industrial y con el $27 \%$ de los puestos de trabajo generados en la industria manufacturera. Incluso, el comportamiento de la tasa de ocupación laboral del parque industrial Jilotepec fue marginalmente mayor respecto a la presentada en el municipio del mismo nombre, razón de considerarla como una de las 
principales actividades alternas que sostiene la economía local, además de que dicho parque está en crecimiento.

Por otro lado, están aquellos parques que perdieron dinamismo, tales como los de Atlacomulco, Toluca, Tultitlán y Cuautitlán Izcalli, donde el desempeño ocupacional de todo el sector descendió, pues como espacios maduros y saturados, las empresas ya no pudieron crecer más de lo que el espacio disponible les permitía, provocando incluso salida de algunas empresas (fuera del parque o incluso del municipio), lo que repercutió en la disminución de empleo industrial, como sucedió en el parque micro industrial Cuautitlán Izcalli. Este hecho también fue explicado por la reconversión productiva, es decir, varias empresas tecnificaron sus procesos de producción, lo que implicó desplazamiento de fuerza de trabajo, como lo que sucedió en Toluca o Lerma. La otra posibilidad fue lo que experimentó el parque de Atlacomulco, donde se quedaron sólo las áreas administrativas y la parte productiva se movió fuera del lugar, provocando una disminución del empleo local.

\section{CONCLUSIONES}

Las políticas públicas y los diversos programas de desarrollo industrial como los incentivos y subsidios fiscales y administrativos, constituye uno de los principales mecanismos para fomentar el establecimiento de nuevas empresas en diversas localidades, así como buscar su descentralización, tal como sucedió con el Estado de México, que pronto se convirtió en una de las entidades más industrializadas del país. Parte de las acciones gubernamentales en la entidad mexiquense fue la implementación de múltiples programas para la construcción de infraestructura, principalmente medios de comunicación, lo que permitió una mayor conectividad entre municipios y regiones, así como el incremento de flujo de mercancías y recursos hacia los diversos mercados del país, buscando su integración a la dinámica económica e industrial que estaba experimentando la región centro y norte del país.

Parte de las acciones de la descentralización industrial fue la construcción de varios corredores industriales, como la ubicada en la zona norte de la entidad, conformado por los municipios de El Oro, Atlacomulco, Jilotepec, Jocotitlán e Ixtlahuaca, cuyo objetivo principal fue propiciar un desarrollo regional convergente a través de su expansión territorial. La

ISSN: 1390-9320, Vol. 6, No. 5, octubre 2019 
construcción de estos parques industriales, se concibió como un mecanismo de articulación del ambiente urbano-regional con las políticas de transporte, desarrollo de recursos naturales, fuerza de trabajo, migración, mercados, energéticos y dotación de infraestructura, así como de convergencia territorial, mediante una mayor vinculación entre los diversos municipios del estado.

A pesar de los esfuerzos público-privado de expansión, ordenación y descentralización de la industria vía parques industriales, se acentuó la heterogeneidad territorial a nivel regional, explicada por la reconcentración de los parques industriales en determinados municipios. Durante el año 2018, el 62\% de estos se concentraron al nororiente y norponiente (conurbados a la Ciudad de México); el 28\% se distribuyó en la región centro, y el restante $10 \%$ en la zona poniente del Estado de México, donde se ha formado el corredor industrial periférico norte.

Este fenómeno dio origen a las denominadas regiones de reconcentración industrial (RRI), que es una clara expresión de la ineficacia de la intervención estatal y el bajo nivel de atracción del desarrollo creados, explicado por la disponibilidad insuficiente de infraestructura y equipamiento urbano industrial, que inhibe el establecimiento de nuevas empresas en dichos polígonos productivos. Dado este bajo nivel de atracción empresarial, no sólo pone en entredicho las acciones gubernamentales, sino los beneficios (en términos laborales) que tienden a opacarse por los impactos negativos que generan dichos desarrollos, tales como el cambio de uso de suelo, el encarecimiento del agua potable, el deterioro de vialidades primarias y secundarías, la generación de contaminación ambiental (ruido frecuente, emisión de bióxido de carbono, incremento de residuos sólidos sobre las vialidades que conducen a los parques industriales, desecho incontrolado de aguas residuales, incremento de tráfico vehicular), así como inseguridad local.

Esta dinámica productiva regional propiciada por los parques industriales, también es reflejo de que las políticas macroeconómicas, como directrices impositivas, no siempre son compatibles con los entornos locales y regionales, más aun, su adopción idealizada puede llevar a generar externalidades negativas y polarizaciones territoriales como sucede en el Estado de México, en donde la convergencia social se desvanece producto de la reconcentración en determinados municipios como los conurbados a la ZMCM y ZMCT.

ISSN: 1390-9320, Vol. 6, No. 5, octubre 2019 


\section{REFERENCIAS BIBLIOGRÁFICAS}

Alonso, L. F. (1991). Las nuevas formas del espacio productivo y sus implicaciones urbanísticas. Geometría. (No. 11): 1991, pp. 2-15.

Asociación Mexicana de Parques Industriales Privados (AMPIP) (2010). Distribución nacional de parques industriales. Recuperado el: 05 de diciembre de 2010. En www.naiopnm.org/2009

Bejar, R. y Casanova, F. (1970). Historia de la industrialización en el estado de México. México: Biblioteca enciclopédica del Estado de México.

Bengston, N. y Van Royen W. (1956). Fundamentals of economic geography. England: Prentice Hall.

FIDEPAR (2009). Directorio de empresas de los parques industriales del estado de México. Recuperado el: 13 de marzo de 2009. Recuperado de http://www.edomex.gob.mx/portal/page/portal/fidepar/dirindustrial

GEM (1963). Sexto informe de gobierno, Gustavo Baz Prada. México: Gobierno del Estado de México.

GEM (1951). Compilación de informes de gobierno, 1946-1950, Alfredo del Mazo Vélez. México: Gobierno del Estado de México.

INEGI (1999). Parques, ciudades y corredores industriales de México. Resultados oportunos. Censos económicos 1999, enumeración integral. México: INEGI.

Lösch, A. (1954). The economics of location. Massachusetts: Yale University Press.

Martínez, M. (1985). Industrialización en México. Hacia un análisis crítico. México: El Colegio de México.

ONUDI (1979). Pautas para el establecimiento de parques industriales en los países en desarrollo. New York: ONU.

Rozga, R. (1992). Las industrias modernas en México; una prueba de conceptualización y clasificación. Recuperado el: 16 de octubre de 2015.

ISSN: 1390-9320, Vol. 6, No. 5, octubre 2019 
Recuperado de https://books.google.com.mx/books?id=KeJx_kgVD$c C \& p g=P A 36 \& l p g=P A 36 \& d q=R .+$ Rozga:+Las+industrias+modernas+en+M\%C3\% A9xico\&source

Rozga, R. e Iglesias, D. (2004). El proceso de industrialización en el Estado de México: tendencias y estado actual. La economía del Estado de México, hacia una agenda de investigación. México: El Colegio Mexiquense, A. C. P pp. 73-102

Secretaría de Economía (SE) (2015). Norma oficial mexicana de parques industriales, versión 2015. NMX-R-046-SCFI-2015. México: Gobierno de la República.

Secretaría de Economía (SE) (2005). Norma oficial mexicana de parques industriales, versión 2005. NMX-R-046-SCFI-2005. México: Gobierno de la República

Sistema Mexicano de Promoción de Parques Industriales (SIMPPI) (2010). Localización e infraestructura de los parques industriales en México. Recuperado el: 18 de agosto de $2017 . \quad$ Recuperado de http://www.contactopyme.gob.mx/parques/intranets.asp.

Weber, A. (1929). Alfred Weber's theory of the location of industries. USA: Chicago University Press.

ISSN: 1390-9320, Vol. 6, No. 5, octubre 2019 